\title{
Renal affectation in polyrheumatoid arthritis
}

Delia TIMOFTE ${ }^{1}$, Adina MANDITA ${ }^{2}$, Andra-Elena BALCANGIU-STROESCU ${ }^{1,3}$, Daniela BALAN ${ }^{3}$, Laura RADUCU ${ }^{4,5}$, Maria Daniela TANASESCU ${ }^{6,7}$, Alexandru DIACONESCU ${ }^{1}$, Dragos DORIN ${ }^{6,7}$, Cristina-lleana $\operatorname{COSCONEL}^{8}$, Dorin IONESCU ${ }^{6,7}$

${ }^{1}$ Department of Dialysis, Emergency University Hospital, Bucharest, Romania

${ }^{2}$ Dialysis Center Sema Parc Bucharest, Bucharest, Romania

${ }^{3}$ Faculty of Dental Medicine, Discipline of Physiology,

"Carol Davila" University of Medicine and Pharmacy, Bucharest, Romania

"Department of Plastic and Reconstructive Surgery, "Prof. Dr. Agrippa Ionescu" Clinical

Emergency Hospital, Bucharest, Romania

${ }^{5}$ Discipline of Plastic and Reconstructive Surgery,

"Carol Davila" University of Medicine and Pharmacy, Bucharest, Romania

${ }^{6}$ Department of Nephrology, Emergency University Hospital, Bucharest, Romania

${ }^{7}$ Department of Medical Semiology, Discipline of Internal Medicine I and Nephrology,

"Carol Davila" University of Medicine and Pharmacy, Bucharest, Romania

${ }^{8}$ Faculty of Dental Medicine, Discipline of Foreign Languages,

"Carol Davila" University of Medicine and Pharmacy, Bucharest, Romania

\begin{abstract}
Polyrheumatoid arthritis (PA) is a chronic systemic inflammatory condition of unknown origin and autoimmune pathogenesis. It affects several articulations, which become damaged and deformed; furthermore, multiple systemic manifestations are often encountered. Rheumatoid arthritis $(R A)$ is an important risk factor for the occurrence of CKD and is mandatory that clinicians possess a good knowledge of the mechanisms determining renal impairment and means to prevent it.
\end{abstract}

Keywords: polyrheumatoid arthritis, CKD, kidney lesions

Chronic kidney disease (CKD) is caused by factors such as high blood pressure, diabetes, glomerulopathies, urinary obstructions of various types [1]. We also highlight that chronic kidney disease also occurs secondary to systemic ailments, including SEL, RA, scleroderma, etc. [2]. The importance of correct management of patients with CKD results from the fact that its occurrence increases the risk of death of the patient [3].
Patient survival with CKD is influenced by a number of associated clinical conditions. This category includes cardiovascular impairment commonly found in this patient population. The frequency of cardiovascular disease increases even more in patients undergoing renal replacement therapy [4].

In chronic hemodialysis patients, the important status is also important for patient survival. An in- 
creased level of inflammation, commonly identified in patients with stage 5 CKD (End-Stage Renal Disease - ESDR), is an important trigger for lowering survival of patients [5]. In diabetic patients, literature studies increasingly describe the association between oxidative stress and microvascular complications, including diabetic kidney disease [6]. The occurrence of nephropathy in diabetic patients is associated with lower life expectancy and an increased mortality risk [7]. To improve the prognosis of these patients, proper management including pharmacological and non-pharmacological treatment (diet) is very important for their survival [8].

Rheumatoid arthritis (RA), being an important risk factor for the occurrence of CKD, requires clinicians a good knowledge of the mechanisms for determining renal impairment and means to prevent it.

The RA etiology is unknown, yet many pathogenic factors, such as genetic factors, environmental ones (different infectious agents, smoking, sex, diet), the proinflammatory cytokines, innate and acquired immune mechanisms, are taken into consideration [9].

RA is characterized by the alteration of the immune mechanisms and an aberant immune response, generating an increased number of autoantibodies (auto-AB); the rheumatoid factor $(R F)$ and the cyclic anti- citrulinated $A B$ proteins (ABAP).

The rheumatoid factor is present in $75-80 \%$ of the patients suffering from RA and has elevated titres in cases with extra-articular manifestations and severe forms of the disease. RF is represented by auto-AB aiming at the FC section of $G$ immunoglobulines, which link the immune complexes [9].

Kidney lesions secondary to the disease or to the specific treatment may occur $[10,11]$. (Table 1).

TABLE 1. Kidney lesions in rheumatoid polyarthritis

\begin{tabular}{|l|}
\hline Renal affectation secondary to the disease \\
\hline Glomerular lesions \\
\hline Amyloidosis \\
\hline Vascular lesions \\
\hline Tubulointers lesiøns \\
\hline Renal affectation secondary to medication \\
\hline Nonsteroidal an tory drugs \\
\hline Analgesics \\
\hline Gold salts \\
\hline Penicillamine \\
\hline Cyclosporine \\
\hline Methotrexate \\
\hline
\end{tabular}

The most frequent types of renal affectation associated to RA are: glomerulonephritis (mem- branous, mesangioproliferative) in $60-65 \%$ of the cases, secondary amyloidosis - 20-30\% of the cases- followed by chronic interstitial nephropathy, rheumatoid vasculitis, analgesic nephropathy, IgA nephropathy and glomerulohephritis with minimal lesions (not very common) $[12,13]$. IgA glomerulonephrites during the treatment with leflunomid and TNF receptor blockers - etanerecep have been reported in rare cases [14].

\section{RENAL AFFECTATION SECONDARY TO RHEUMATOID ARTHRITIS}

\section{1.a. Glomerular lesions}

Mesangial glomerulonephritis is encountered in $35-78 \%$ of the PA patients with renal affectation, especially those who have had the disease for a long time (12.9 \pm 10.4 years). Interleukine 6 , which has a role in mesangial cells increase, was involved in this type of nephropathy.

Glomerular lesions occur as a result of the deposition of $A$ and $M$ immunoglobulins in the mesangium. Inflammation occurs at this level and the complement is activated on alternating pathways. The clinical picture presents nephritic syndrome and approx. $1 \mathrm{~g} / 24 \mathrm{~h}$ proteinuria and microscopic hematuria [15].

Membranous glomerulonephritis is uncommon in the absence of the treatment with gold salts and $d$-penicillamine. The lesions occur due to the build-up of circulatory immune complexes, IgG immunoglobulins and the C3 faction of the segmented and diffuse complement at the level of the glomerular basal membrane, which thickens. Sometimes severe, or merely non selective, isolated proteinuria is found paraclinically at nephrotic level. Microscopic hematuria can occur in some cases.

This type of glomerular affectation is more frequently associated to RA therapy with penicillamine and gold salts, and the discontinuation generally determines the remission of the nephrotic syndrome. The incidence of proteinuria increases proportionally with the length of the therapy with gold salts, but the severity of the proteinuria does not depend on the total gold dosage.

Rare cases of IgA nephropathy and disease with minimal lesions have been described.

\section{1.b. Secondary amyloidosis}

Secondary amyloidosis (AA) occurs in $3-10 \%$ of PA cases, especially in cases with lengthy disease, with high titres of the rheumatoid factor. It is characterized by the serum amyloid A deposits, 
produced in the liver following chronic inflammation [15]. Under the optic microscope, the amyloid deposits are mesangial initially and later become peritubular and perivascular.

Clinically, it manifests by sometimes severe proteinuria with nephrotic syndrome, without hyperlipidemia and poor urinary sediment. The renal amyloidosis progresses to renal failure and to uremia. Approximately $20 \%$ of the patients require substituting treatment to renal functions.

AA amyloidosis has a severe prognosis, with frequent infections and venous thrombosis secondary to the nephrotic syndrome; complications are more frequent and severe in elderly patients, with high persistent serum amyloid $A$ titres and advanced renal failure at the time they are diagnosed [16].

A survey which studied 374 patients with secondary AA amyloidosis for 86 months, showed that the renal affectation was the chief manifestation of the disease, and mortality correlated with the serum amyloid A concentration; the highest mortality was in patients with serum amyloid $A$ level $>155 \mathrm{mg} / \mathrm{l}$. A favorable evolution was found in the group of patients with serum amyloid $A<4$ $\mathrm{mg} / \mathrm{l}$ [17].

A study undertaken in Japan on RA patients found that the treatment of the main disease can determine the decrease of the amyloid deposits, of the proteinuria, along with the improvement of the renal function and increased survival [18].

The survival rate of patients on dialysis is low because of malnutrition and the vascular and infectious complications.

In $20-30 \%$ of the cases of renal transplants, the AA amyloidosis can relapse on the transplanted kidney [4].

\section{1.c. Vascular lesions}

Rheumatoid vasculitis in PA can affect different blood vessels, both small/medium-size arteries and post capillary venules, with tissue ischemia and vascular obstructions. The vascular lesions are more common in patients with long articular affectation and articular destruction, with rheumatoid nodules and increased serum titres of the rheumatoid factor. A picture similar to panarteritis nodosa, with the involvement of the large vessels and rapid evolution towards renal failure can occur in rare cases.

\section{1.d. Tubulointerstitial lesions}

Are more common in Sjögren syndrome secondary to PA (xerostomia, xerophtalmia, sicca keratoconjunctivitis in $10-30 \%$ of the cases) and in patients treated with aspirin and phenacetine. Interstitial nephritis is characterized by an interstitial lymphocytic infiltrate accompanied by tubular atrophy and interstitial fibrosis.

Clinically, it manifests by:proteinuria, hematuria, renal failure [15].

\section{RENAL AFFECTATION SECONDARY TO MEDICATION}

\section{2.a. Nonsteroidal anti-inflammatory drugs (NSAID)}

NSAIDs can induce acute nephritis, probably through immunologic mechanism, or can lead to a decrease in the glomerular renal flow, following the inhibition of the renal prostaglandins synthesis; the outcome is secondary nitrate retention, reversible when medication is discontinued.

Elderly patients with hypovolemia and pre-existing renal impairment are more susceptible.

\section{2.b. Analgesic nephropathy has been associated to the treatment with phenacetine and manifests by interstitial nephritis and papillary necrosis.}

\section{2.c. Renal lesions induced by the gold salts treatment}

The secondary effects of the gold salts therapy generally occur after 3-6 months of treatment and, besides renal affectation, stomatitis lesions, cutaneous eruptions, medullary depression may occur.

The renal lesions following the gold salts therapy, as described histologically, were: membranous glomerulonephritis in $75 \%$ of the cases, the increase of the mesangial matrix in $20 \%$ of the cases and glomerulonephritis with minimal lesions in about $5 \%$ of the patients [10]. Gold salts can build up in the proximal or distal tubes, as well as at the level of the renal interstitium.

Proteinuria is the most common sign of renal affectation and is present in approximately $25 \%$ of the cases, and in a third of these is at nephritic level. The proteinuria frequency increases with the length in time of the therapy with gold salts and generally appears 4-6 months after the treatment started. It may associate microscopic hematuria.

HLA-B8 and DR3 antigens have been more frequently associated to the occurrence of renal lesions following the gold salts therapy, possibly because of the tendency to form antibodies more easily [10]. 


\section{2.d. Renal lesions induced by the treatment with D-penicillamine}

Penicillamine produces a nephropathy similar to the one caused by the gold salts, which manifests by proteinuria with nephrotic syndrome in a third of the patients. It can associate to microscopic hematuria.

On average, these changes occur after 8 months of treatment and correlate with the administered doses. The incidence of renal lesions was lower when doses were smaller.

\section{2.e. Renal lesions induced by the treatment with cyclosporine}

Cyclosporine cause renal affectation through mechanisms, which are not fully explained and produce intra-renal vasoconstriction followed by the decrease in glomerular filtration and nitrate retention, along with interstitial nephritis. The renal effects correlate with serum doses over 200 $\mathrm{mg} / \mathrm{ml}$.

The doses of cyclosporine used in rheumatoid polyartitis are between 2.5 and $10 \mathrm{mg} / \mathrm{kgb} /$ day; higher doses cause the increase of serum creatinine in $60 \%$ of the patients [10]. The cyclosporine doses must be adjusted according to the serum concentration $(<150 \mathrm{ng} / \mathrm{ml})$ [15].
The above data emphasize the need to adapt the dose of cyclosporine to the level of renal failure and to avoid the association with further nephrotoxic drugs and NSAIDs.

\section{2.f. Renal lesions induced by the treatment with methotrexate}

Methotrexate is a folic acid analogue, with a significant anti-inflammatory effect by decreasing the TNF alpha secretion (alpha factor of tumor necrosis) and is the first treatment choice in active rheumatoid polyarthritis [19]. It is eliminated unchanged, mainly through the renal pathway.

The common doses are 7.5-5 mg/week and renal affectation is rare and reversible when medication is discontinued [20].

The treatment of the renal lesions in rheumatoid polyarthritis involves first the discontinuation of the medication which triggered the renal dysfunction, suitable water intake, avoidance of hypotension and other nephrotoxic drugs, and in case of severe renal affectation treatment for substituting renal functions (dialysis).

\section{Acknowledgement}

All authors have contributed equally to the present work and thus are main authors.

\section{Conflict of interest: none declared Financial support: none declared}

\section{REFERENCES}

1. Tănăsescu MD, Balcangiu Stroescu $A E$, Răducu L, Limbău AM, Diaconescu AC, Bălăn DG, lonescu D. Uterine cervical cancer and chronic kidney disease an association that should not be skipped. Rev. Chim. (Bucharest), 69, No.9, 2018, pp. 2524-2526.

2. Anders HJ, Vielhauer V. Renal comorbidity in patients with rheumatic diseases. Arthritis Res Ther. 2011; 13(3): 222.

3. Tonelli M, Wiebe N, Culleton B, House A, Rabbat C, Fok M, McAlister F, Amit X. Chronic Kidney Disease and Mortality Risk: A Systematic Review. JASN July 2006, 17 (7) 2034-2047

4. Balcangiu Stroescu $A E$, Tănăsescu MD, Diaconescu AC, Răducu L, Constantin AM, Bălan DG, Tărmure V, lonescu D. Cardiovascular comorbidities, inflammation and serum albumin levels in a group of hemodialysis patients. Rev. Chim. (Bucharest), 69, No. 4, 2018, pp. 926-929.

5. Balcangiu-Stroescu $A E$, Tănăsescu $M D$, Diaconescu AC, Răducu L, Bălan DG, Ionescu D. A brief presentation of the characteristics of hemodialysis membranes. Materiale Plastice, 55, No.3, 2018, pp. 332-334.

6. Totan A, Balcangiu-Stroescu AE, Meleșcanu Imre M, Miricescu D, Bălan D, Stănescu II, lonescu D, Timofte D, Tănăsescu MD, Greabu M, XOR. Possible
Correlations with Oxidative Stress and Inflammation Markers in the Context of Diabetic Kidney Disease. Rev. Chim. (Bucharest), 70, No.4, 2019, pp. 1396-1398.

7. Balcangiu-Stroescu $A E$, Tănăsescu MD, Diaconescu AC, Răducu L, Bălan DG, Mihai A, Tănase M, Stănescu II, lonescu D. Diabetic nephropathy: A concise assessment of the causes, risk factors and implications in diabetic patients. Rev.Chim. (Bucharest), 69, No.11, 2018, pp. 3118-3121.

8. Bălan DG, Balcangiu-Stroescu $A E$, Tănăsescu MD, Diaconescu $A C$, Răducu L, Andrada Mihai, Tănase M, Stănescu II, lonescu D. Nutritional intervention in patients with diabetic renal disease - a brief presentation. Revista de Chimie.(Bucharest), 69, No.11, 2018, pp. 3178-3182.

9. Bălănescu AR. Immune mechanisms in rheumatology. Ed. Medicala, 2019, pp. 127-156.

10. lonescu D, Popescu A. Renal lesions associated to the therapy with gold salts, penicillamine and cyclosporine in rheumatoid polyarthritis. The Journal of Rheumatology, Vol VIII no 1, 2000, pp. 45-46.

11. Covic A. Nephrology. Theoretical and practical principles. Demiurg Printing House, 2018, pp. 337-338.
12. Icardi A et al. Kidney involvement in rheumatoid arthritis. Reumatismo, 2003;55: pp. 76-85.

13. Rodriquez T. Renal complications in rheumatoid arthritis: Evolution and management. Rheumatology Advisor, December 20, 2018.

14. Mori $S$ et al. Prevalence of and factors associated with renal dysfunction in rheumatoid artheritis patients: A crosssectional study in community hospitals. Clin. Rheumathologogy 2017, 36: pp. 2673-2682.

15. Gherasim I et al. Diseases of the renal system, in Treatise of Internal Medicine, vol IV, Ed. Medicala, 2002, pp.351-355.

16. Mircescu $G$ et al. Glomerulopathies. Ed. Medicală, 2017, pp. 87-88; 179-185; 279-295

17. Lachmann HJ et al. Natural History and Outcome in Systemic AAAmyloidosis. The New England Journal of Medicine, 2007, 356: pp. 2361- 2371.

18. Nakamura T. Clinical strategies for amyloid A amyloidosis secondary to rheumatoid arthritis. Journal Modern Rheumatology, vol.18, 2008 pp. 109-118.

19. Ungureanu G, Covic A. Medical therapeutics. Ed. Polirom, 2014, p. 685.

20. Stroescu V. Pharmacological bases of medical practice. Ed. Medicală, 1998, p. 1176. 